\title{
Comparison of the prognostic value of platelet-based prognostic models in patients with malignant hepatic tumors after TACE therapy ${ }^{1}$
}

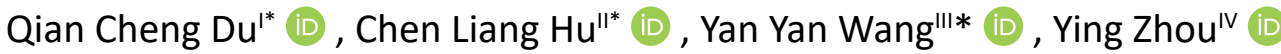

' Master, Department of General Surgery, Shanghai Fourth People's Hospital Affiliated to Tongji University School of Medicine, Shanghai, China. Intellectual, scientific, conception and design of the study; acquisition, analysis and interpretation of data; technical procedures; statistical analysis; manuscript preparation, final approval.

" Master, Department of Hepatopancreatobiliary Surgery, the Affiliated Hospital of Qinghai University, Xining, China. Intellectual, scientific, conception and design of the study; acquisition, analysis and interpretation of data; technical procedures; statistical analysis; manuscript preparation, final approval.

II'Master, Department of Hematology, Affiliated Fuyang Hospital of Anhui Medical University, Fuyang, China. Intellectual, scientific, conception and design of the study; acquisition, analysis and interpretation of data; technical procedures; statistical analysis; manuscript preparation, final approval.

IV Full Professor, Department of Hepatopancreatobiliary Surgery, the Affiliated Hospital of Qinghai University and Qinghai Province Key Laboratory of Hydatid Disease Research, Qinghai University, Xining, China. Acquisition, analysis and interpretation of data; technical procedures; critical revision; manuscript preparation, final approval.

*Contributed equally to this work.

\begin{abstract}
Purpose: To investigate the prognostic value of 17 platelet-based prognostic scores in patients with malignant hepatic tumors after TACE therapy.

Methods: In total, 92 patients were divided into death group and survival group according to long-term follow-up results. The AUC was calculated to determine the optimal cut-off values for predicting prognosis. To determine better prognostic models, platelet-based models were analyzed separately after being showed as binary according to cut-off values. Cumulative survival rates of malignant hepatic tumors were calculated using Kaplan-Meier curves and differences were analyzed by the log-rank test. Univariate and multivariate analyses were performed to identify platelet-based prognostic scores associated with overall survival.

Results: Univariate analysis showed that APGA, APRI, FIB-4, FibroQ, GUCl, King's score, Lok index, PAPAS, cirrhosis, number of tumors, vascular cancer embolus, AFP, ALP and APTT were significantly related to prognosis. A multivariate analysis showed that the APGA, number of tumors, ALP and APTT were independently associated with overall survival.

Conclusion: This study showed that the APGA, a platelet-based prognostic score, was an independent marker of prognosis in patients with malignant hepatic tumors after TACE and was superior to the other platelet-based prognostic scores in terms of prognostic ability.
\end{abstract}

Key words: Transcatheter arterial chemoembolization. Blood Platelets. Liver Neoplasms. Prognosis. 


\section{- Introduction}

Malignant hepatic tumors were the sixth most frequently diagnosed cancer worldwide, and the fourth leading cause of tumor-related deaths ${ }^{1}$. Patients with malignant hepatic tumors were treated by different methods based on TNM stage of tumor and hepatic function reserve before accepting treatment. Although the prognosis of malignant hepatic tumors had improved significantly, the prognosis was still unsatisfactory with a 5-year survival rate of about $5 \%-6 \%^{2}$. There were no obvious symptoms at the early stage, and most of the patients had entered the middle and late stage at the time of diagnosis. Patients with intermediate or advanced stage tumors were not suitable for radical treatment according to the Barcelona Clinic Liver Cancer staging system (BCLC) ${ }^{3}$. Transcatheter arterial chemoembolization (TACE) was the optimum palliative treatment for patients with unresectable malignant hepatic tumors. The median survival time of patients with malignant hepatic tumors after TACE was 12 months, which was significantly lower than that after radical liver resection ${ }^{4}$. Therefore, it is very important to evaluate the prognosis of patients performing TACE according to specific laboratory indexes before treatment.

The main functions of platelets (PLT) were hemostasis and thrombosis. Recently, many reports showed that platelets and platelet-activation molecules played an important part in tumor cell growth, metastasis, and angiogenesis ${ }^{5-8}$. Non-invasive predicting models based on laboratory tests had been widely used, and could be a feasible method for evaluating the extent of hepatic fibrosis, tumor recurrence and so on. The aspartate aminotransferase (AST)-to-platelet ratio index (APRI), the King's Score, the FibroQ and other models had been proposed to predict hepatic fibrosis, liver cirrhosis and liver dysfunction in patients with chronic hepatitis ${ }^{9-11}$. Hepatitis, cirrhosis and liver cancer were the three stages of disease development; however, it was not clear whether these models could predict the prognosis of malignant liver tumor. Maeda et al. ${ }^{12}$ described that Fibrosis-4 (FIB-4) index could evaluate the recurrence rate and 5-year recurrence rate after hepatectomy in patients with hepatocellular carcinoma. However, the non-invasive models based on tumor clinical characteristics and peritumoral fibrosis for predicting prognosis of patients accepting TACE were still rare, especially in overall survival.

We aimed to investigate the prognostic value of these platelet-based prognostic models in patients with malignant hepatic tumors who underwent TACE, and to select the most predictive model for guiding TACE preoperative evaluation. Thus, a retrospective analysis of 92 patients who had received TACE was performed.

\section{- Methods}

In total, 92 patients with intermediate or advanced diagnosed malignant hepatic tumors and performing TACE that had been treated at the Intervention Department, the Affiliated Hospital of Qinghai University, between November 2011 and October 2018 were enrolled in the study. Comprehensively baseline information, including clinical, laboratory, imageological and follow-up data was available for all patients. Patients with coexistent hematologic diseases, patients who had received blood transfusion within the previous 6 months, extrahepatic tumor, and incomplete data were excluded. Patients were followed after TACE treatment every 3-6 months until death or dropout. This study was approved by the Ethics Committee at the Qinghai University Affiliated Hospital and complies with the provisions of the Declaration of Helsinki.

The diagnosis of malignant hepatic tumors should be based on imageological techniques obtained by dynamic contrast-enhanced computed tomography (CT), dynamic contrast-enhanced magnetic resonance imaging (MRI), and the typical peripheral blood tumor markers of hepatic tumors ${ }^{13}$. Tumor-related parameters such as the maximal diameter of tumor, the number of tumors, vascular invasion, vascular cancer embolus, the diameter of spleen and extrahepatic metastases were evaluated by imageological techniques. All peripheral blood parameters were derived from blood draws taken within 7 days before the first TACE.

\section{Platelet-based prognostic scores and other variables}

Electronic medical records were used to obtain relevant information, including age, sex, monocyte fraction, neutrophil fraction, levels of alanine aminotransferase (ALT), AST, total cholesterol (TCH), alkaline phosphatase (ALP), $\gamma$-glutamyl transpeptidase (GGT), PLT, prothrombin time (PT), activated partial thromboplastin time (APTT), international normalized ratio (INR), alpha-fetoprotein (AFP), etiology (HBV or HCV), ascites, cirrhosis status, number of tumors, diameter of maximal tumor, vascular invasion, vascular cancer embolus, diameter of spleen, extrahepatic metastases and concomitant disease. The primary 
outcome measure for the study was survival status (death or survival).

The Pohl score ${ }^{14}$, aspartate aminotransferase/ alanine aminotransferase ratio-platelet count score $(A A R P)^{15}$, age/ platelet count index $(A P I)^{16}$, cirrhosis discriminant score $(\mathrm{CDS})^{17}, \mathrm{APRI}{ }^{9}, \mathrm{FIB}-4^{18}{ }^{18}$ FibroQ $^{11}$, Fibrosis index based on the three factors (Lok index $)^{19}$, Goteborg University cirrhosis index $(\mathrm{GUCl})^{20}$, aspartateaminotransferase/ platelet count/ $\mathrm{\gamma}$-glutamyl transpeptidase/ alphafetoprotein index $(A P G A)^{21}$, platelet count/ age/ ALP/ AFP/ AST index (PAPAS) ${ }^{22}$, fibrosis index based on the four factors (King's score) ${ }^{10}$, $\gamma$-glutamyl transpeptidase/ platelet count ratio index $(\mathrm{GPR})^{23}$, $\mathrm{Y}$-glutamyl transpeptidase/ platelet count/ serum albumin index (S-index ${ }^{24}$, platelet count/ spleen diameter $(\mathrm{mm})$ ratio index (PSR) ${ }^{25}$, monocyte fraction/segmented neutrophil fraction/ platelet count index (P2/MS) ${ }^{26}$ and Platelet count/ $\mathrm{Y}$-glutamyl transpeptidase/ age/ cholesterol index (Forns index) ${ }^{27}$ were constructed as described in Table 1.

Table 1 - Scoring of platelet-based models.

\begin{tabular}{|c|c|}
\hline Index & Formulas \\
\hline Pohl score & 1: AAR $>1$ and PLT $<150 \times 10^{9} / \mathrm{L}$ or else, the score $=0$ \\
\hline AARP & 1: $\mathrm{AAR}>1$ or $\mathrm{PLT}<150 \times 10^{9} / \mathrm{L}$ or else, the score $=0$ \\
\hline API & $\begin{array}{c}\text { Age (years) }:<30=0 ; 30-39=1 ; 40-49=2 ; 50-59=3 ; 60-69=4 ; \geq 70=5 . \text { PLT: } \geq 225=0 ; 200-224 \\
=1 ; 175-199=2 ; 150-174=3 ; 125-149=4 ;<125=5 \text { API is the sum of age and platelet scores and } \\
\text { therefore varied from } 0-10\end{array}$ \\
\hline CDS & $\begin{array}{c}\text { PLT: }>340=0 ; 280-339=1 ; 220-279=2 ; 160-219=3 ; 100-159=4 ; 40-99=5 ;<40=6 \text { ALT/AST } \\
\text { ratio: }>1.7=0 ; 1.2-1.7=1 ; 0.6-1.19=2 ;<0.6=3 \text { INR: }<1.1=0 ; 1.1-1.4=1 ;>1.4=2 \text { CDS is the sum } \\
\text { of the above }\end{array}$ \\
\hline APRI & [AST $(/$ ULN $) \times 100] /$ PLT $\left(10^{9} / \mathrm{L}\right)$ \\
\hline FIB-4 & [age $($ years $) \times$ AST $(U / L)] /\left[\operatorname{PLT}(109 / L) \times \operatorname{ALT}(U / L)^{1 / 2}\right]$ \\
\hline FibroQ & $10 \times($ Age $\times$ AST $\times$ PT INR $/$ ALT $\times$ PLT $)$ \\
\hline Lok index & $\log$ odds $=-5.56-0.0089 \times \operatorname{PLT}\left(10^{9} / \mathrm{L}\right)+1.26 \times$ AAR $+5.27 \times$ INR, lok index $=\exp (\log$ odds $) /[1+\exp (\log$ odds $)]$ \\
\hline GUCI & AST $\times$ INR $\times 100 /$ PLT $\left(10^{9} / L\right)$ \\
\hline APGA & $\begin{array}{c}\log (\text { index })=1.44+0.1490 \times \log [\mathrm{GGT}(\mathrm{U} / \mathrm{L})]+0.3308 \times \log [\mathrm{AST}(\mathrm{U} / \mathrm{L})]-0.5846 \times \log \left[\mathrm{PLT}\left(10^{9} / \mathrm{L}\right)\right]+0.1148 \times \log \\
{[\operatorname{AFP}(\mathrm{ng} / \mathrm{mL})+1)}\end{array}$ \\
\hline PAPAS & $\begin{array}{c}\log (\text { index }+1)=0.025+0.0031[\text { age }(\text { years })]+0.1483 \times \log [A L P(U / L)]+0.004 \times \log [A S T(U / L)]+0.0908 \times \log \\
{[\operatorname{AFP}(n g / m L)+1)-0.028 \times \log \left[P L T\left(10^{9} / L\right)\right]}\end{array}$ \\
\hline King's score & Age $\times$ AST $\times$ INR/PLT $\left(10^{9} / \mathrm{L}\right)$ \\
\hline GPR & GGT/PLT(109/L) \\
\hline S-index & $1000 \times \mathrm{GGT} /\left[\mathrm{PLT}\left(10^{9} / \mathrm{L}\right) \times \mathrm{ALB}^{2}\right]$ \\
\hline PSR & $\operatorname{PLT}\left(10^{9} / \mathrm{L}\right) /$ spleen diameter $(\mathrm{mm})$ \\
\hline $\mathrm{P} 2 / \mathrm{MS}$ & $\operatorname{PLT}^{2}\left(10^{9} / \mathrm{L}\right) /($ monocyte fraction $\times$ segmented neutrophil fraction $)$ \\
\hline Forns index & $7.811-3.131 \times \ln \left[\mathrm{PLT}\left(10^{9} / \mathrm{L}\right)\right]+0.781 \times \ln (\mathrm{GGT})+3.467 \times \ln ($ age $)-0.014 \times($ cholesterol $)$ \\
\hline $\begin{array}{l}\text { AAR, Asparta } \\
\text { platelet coun } \\
\text { Cirrhosis disc } \\
\text { four factors; } \\
\text { University Cir } \\
\text { GGT, v-glutan } \\
\text { index; King's } \\
\text { albumin; S-in } \\
\text { index; P2/MS } \\
\text { transpeptidas }\end{array}$ & $\begin{array}{l}\text { minotransferase/alanine aminotransferase ratio; PLT, Platelet count; AARP, AAR-platelet count score; API, Age/ } \\
\text { dex; ALT, Alanineaminotransferase; AST, Aspartateaminotransferase; INR, international normalized ratio; CDS, } \\
\text { nant score; APRI, Aspartate aminotransferase/ platelet count ratio index; FIB-4, Fibrosis index based on the } \\
\text { rothrombin time; FibroQ, Fibro-quotient; Lok-index, Fibrosis index based on the three factors; GUCI, Goteborg } \\
\text { sis Index; APGA, Aspartateaminotransferase/ platelet count/ } \text {-glutamyl transpeptidase/ alphafetoprotein index; } \\
\text { ranspeptidase; AFP, Alpha-fetoprotein; ALP, Alkalinephosphatase; PAPAS, Platelet count/ age/ ALP/ AFP/ AST } \\
\text { e, Fibrosis index based on the four factors; GPR, v-glutamyl transpeptidase/ platelet count ratio index; ALB, serum } \\
\text { p-glutamyl transpeptidase/ platelet count/ serum albumin index; PSR, platelet count/ spleen diameter (mm) ratio } \\
\text { onocyte fraction/ segmented neutrophil fraction/ platelet count index; Forns index, Platelet count/ p-glutamyl } \\
\text { age/ cholesterol index. }\end{array}$ \\
\hline
\end{tabular}




\section{Treatment and patient's follow-up}

All hypervascular nodules were treated by TACE using oxaliplatin (AiHeng ${ }^{\circledR}$; Jiangsu HengRui pharmaceutical company limited (co. LTD), Jiangsu, China) or a combination of epirubicin (AiDaSheng ${ }^{\circledR}$; Hisun Pfizer pharmaceutical co. LTD, ZheJiang, China) or a combination of pirarubicin (THP®; Shenzhen Wanle pharmaceutical co. LTD, Guangdong, China). Patients also performed super-selective catheterization and underwent an injection of Lipiodol into nutrient artery of the tumor, followed by an injection of Collagen Sponge (Trauer ${ }^{\circledR}$; GuangZhou Trauer Biotechnology Co. LTD, GuangZhou, China). The TACE treatment was repeated every time intrahepatic relapse was found.

Patients were regularly followed at outpatient clinics after discharge, by either CT or MRI, abdominal ultrasound, physical examination, chest radiography, and peripheral blood markers tests (including liver function test and serum AFP). The same evaluations were performed as follow-ups every 3 months for the first year, and every 6 months after the first year. The starting time of follow-up was the date of initial treatment of malignant hepatic tumors. The cut-off time of follow-up was the date of last follow-up (January 2019) or death.

\section{Statistical analysis}

All continuous variables that obey normal distribution were described as the means \pm standard deviation and were compared using the independent t-test. Other continuous variables that do not obey normal distribution were presented as the median and range. Univariate analysis of the prognosis and possible clinical factors for categorical variables were tested by Pearson $x^{2}$ test. The overall survival rates were evaluated using the Kaplan-Meier curve, and differences in the survival rates between the groups were statistically compared by the log-rank test. The receiver operating characteristic curve (ROC) was also calculated and the area under the curve (AUC) was generated to discriminate the ability of each scoring systems. Meanwhile, The ROC could obtain the optimal cut-off point (the sum of specificity and sensitivity is the highest cumulative value) of each variable for overall survival. The multivariate analysis was evaluated for the prognostic factors using the Cox proportional hazard model. A bilateral probability (P) value $<0.05$ was considered statistically significant. All statistical analysis was performed using the IBM SPSS Statistics software package v.23.0 (IBM Corporation, 2015, USA).

\section{- Results}

\section{Patient characteristics}

The baseline characteristics of 92 patients are presented in Table 2. The median age of 92 patients was 58 (range 32-82) years. Sixty-seven (72.8\%) patients were males and $25(27.2 \%)$ patients were females. One $(1.1 \%)$ patient was positive for antibodies to hepatitis C virus (anti-HCV), 52 (56.5\%) patients were positive for hepatitis B surface antigen. Seventy-five patients (81.5\%) had presented normal liver function (Child-Pugh A grade), 49 patients (53.3\%) were diagnosed with cirrhosis, and 9 patients $(9.8 \%)$ presented ascites. All patients were treated by TACE. There was no surgically related mortality. The Pearson $x^{2}$ test indicated that the prognosis of patients with multiple tumor lesions $(p=0.032)$ or presenting vascular cancer embolus $(p=0.025)$ was poor, and there was significant statistical significance. 
Table 2 - Baseline characteristic of included patients.

\begin{tabular}{|c|c|c|c|c|}
\hline \multirow{2}{*}{ Variable } & \multirow{2}{*}{ Total } & \multicolumn{2}{|c|}{ Survival status } & \multirow{2}{*}{ P value } \\
\hline & & Survival & Death & \\
\hline Sex & & & & 0.681 \\
\hline man & 67 & 37 & 30 & \\
\hline women & 25 & 15 & 10 & \\
\hline Hepatitis & & & & 0.208 \\
\hline Yes & 53 & 27 & 26 & \\
\hline No & 39 & 25 & 14 & \\
\hline Ascites & & & & 0.442 \\
\hline Yes & 9 & 4 & 5 & \\
\hline No & 83 & 48 & 35 & \\
\hline Cirrhosis & & & & 0.256 \\
\hline Yes & 49 & 25 & 24 & \\
\hline No & 43 & 27 & 16 & \\
\hline Child-Pugh classification & & & & 0.195 \\
\hline A & 75 & 40 & 35 & \\
\hline B & 17 & 12 & 5 & \\
\hline Polycythemia & & & & 0.578 \\
\hline Yes & 3 & 1 & 2 & \\
\hline No & 89 & 51 & 38 & \\
\hline \multicolumn{5}{|l|}{ Number of tumors } \\
\hline single & 53 & 35 & 18 & 0.032 \\
\hline multiple & 39 & 17 & 22 & \\
\hline Vascular cancer embolus & & & & 0.025 \\
\hline Yes & 16 & 5 & 11 & \\
\hline No & 76 & 47 & 29 & \\
\hline Age (year) & $57 \pm 9$ & $55 \pm 9$ & $58 \pm 10$ & 0.240 \\
\hline Diameter of spleen $(\mathrm{mm})$ & $107.25(77-194.1)$ & $107.25(83.90-194.1)$ & $106.45(77-175.3)$ & 0.795 \\
\hline $\operatorname{AFP}(\mathrm{ng} / \mathrm{ml})$ & $461.94(0.83-2000)$ & $238.64(0.83-2000)$ & $728.20(1.76-2000)$ & 0.130 \\
\hline ALB $(g / L)$ & $36.09 \pm 5.62$ & $37.60 \pm 5.77$ & $35.50 \pm 5.52$ & 0.356 \\
\hline $\mathrm{TCH}(\mathrm{mmol} / \mathrm{L})$ & $3.62(1.84-8.10)$ & $3.59(1.94-7.42)$ & $3.89(1.84-8.10)$ & 0.503 \\
\hline $\operatorname{ALT}(U / L)$ & $45(7-169)$ & $44.5(14.4-169)$ & $45.5(7-120.7)$ & 0.925 \\
\hline AST $(U / L)$ & $53(13-438)$ & $51(13-438)$ & $58(16-188)$ & 0.262 \\
\hline $\operatorname{ALP}(U / L)$ & $145.50(63-1854.5)$ & $141.5(67.1-908)$ & $149.85(63-1854.5)$ & 0.584 \\
\hline GGT (U/L) & $153.65(12-1257)$ & $153.15(19-1257)$ & $152(12-622.1)$ & 0.598 \\
\hline APTT (s) & $33.38 \pm 6.45$ & $32.42 \pm 6.70$ & $34.53 \pm 5.94$ & 0.119 \\
\hline PT (s) & $12.9(9.9-17.70)$ & $12.8(9.9-17.7)$ & $13.45(11-17.4)$ & 0.464 \\
\hline INR & $1.08(0.83-1.50)$ & $1.07(0.83-1.5)$ & $1.12(0.92-1.48)$ & 0.419 \\
\hline
\end{tabular}

AFP, alpha fetoprotein; ALB, albumin; TCH, total cholesterol; ALT, alanine transaminase; AST, aspartate amino transferase; ALP, alkaline phosphatase; GGT, gamma-glutamyl transpeptidase; APTT, activated partial thromboplastin time; PT, prothrombin time; INR, international normalized ratio. 
The basic characteristics obtained by introducing all parameter values into the 17 platelet-based models were showed in Table 3. The Pearson $\mathrm{x}^{2}$ test or Wilcoxon test or independent $\mathrm{t}$-test showed that the Pohl score $(p=0.035), C D S(p=0.006), A P R I(p=0.032)$, King's score $(p=0.015)$, Lok index $(P=0.015)$, FIB-4 $(p=0.010)$, FibroQ $(p=0.008)$ and $\mathrm{GUCl}(\mathrm{P}=0.033)$ were significantly different between survival and death patients.

Table 3 - Characteristics of platelet count and platelet-based models.

\begin{tabular}{|c|c|c|c|c|}
\hline \multirow{2}{*}{ Variable } & \multirow{2}{*}{ Total } & \multicolumn{2}{|c|}{ Survival status } & \multirow{2}{*}{ P value } \\
\hline & & Survival & Death & \\
\hline AARP & & & & 0.447 \\
\hline negative & 12 & 8 & 4 & \\
\hline positive & 80 & 44 & 36 & \\
\hline Pohl score & & & & 0.035 \\
\hline negative & 55 & 36 & 19 & \\
\hline positive & 37 & 16 & 21 & \\
\hline PLT $\times 109 / L$ & $136.50(37-447)$ & $149(38-447)$ & $115.50(37-322)$ & 0.085 \\
\hline API & $7(1-10)$ & $6(2-10)$ & $8(1-10)$ & 0.074 \\
\hline CDS & $6(3-10)$ & $6(3-10)$ & $7(3-9)$ & 0.006 \\
\hline APRI & $1.16(0.16-16.47)$ & $0.975(0.22-16.47)$ & $1.596(0.16-6.6)$ & 0.032 \\
\hline King's score & $27.59(3.68-305.10)$ & $23.27(4.92-305.10)$ & $34.45(3.68-225.60)$ & 0.015 \\
\hline Lok index & $0.63(0.09-0.99)$ & $0.56(0.09-0.97)$ & $0.75(0.19-0.99)$ & 0.015 \\
\hline P2/MS & $49.88(1.05-521.57)$ & $60.56(1.05-521.57)$ & $34.14(1.98-399.04)$ & 0.196 \\
\hline PAPAS & $3.83 \pm 1.26$ & $3.61 \pm 1.12$ & $4.10 \pm 1.39$ & 0.064 \\
\hline PSR & $1.29(0.30-4.04)$ & $1.37(0.30-4.04)$ & $0.99(0.39-3.40)$ & 0.139 \\
\hline S-index & $0.84(0.06-10.9)$ & $0.812(0.10-10.9)$ & $0.892(0.06-4.54)$ & 0.501 \\
\hline FIB-4 & $3.29(0.87-26.59)$ & $2.867(0.91-24.50)$ & $4.905(0.87-26.59)$ & 0.010 \\
\hline FibroQ & $5.66(1.25-45.83)$ & $4.821 .25-45.83)$ & $7.79(1.35-45.12)$ & 0.008 \\
\hline GUCI & $48.96(6.23-663.26)$ & $39.21(8.06-663.26)$ & $62.48(6.23-300.80)$ & 0.033 \\
\hline GPR & $1.08(0.05-13.66)$ & $1.07(0.16-13.66)$ & $1.065(0.05-5.2)$ & 0.671 \\
\hline APGA & $22.76(4.39-68.33)$ & $22.12(6.68-65.83)$ & $26.06(4.39-68.33)$ & 0.097 \\
\hline Forn index & $10.21 \pm 1.91$ & $9.95 \pm 1.88$ & $10.53 \pm 1.94$ & 0.151 \\
\hline
\end{tabular}

AAR, Aspartate aminotransferase/ alanine aminotransferase ratio; AARP, AAR-platelet count score; P2/MS, monocyte fraction/ segmented neutrophil fraction/ platelet count index; APGA, Aspartateaminotransferase/ platelet count/ $\mathrm{Y}$-glutamyl transpeptidase/ alphafetoprotein index; API, Age/ platelet count index; APR, Aspartate aminotransferase/ platelet count ratio index; PSR, platelet count/ spleen diameter $(\mathrm{mm})$ ratio index; CDS, Cirrhosis discriminant score; S-index, $v$-glutamyl transpeptidase/ platelet count/ serum albumin index; Lok-index, Fibrosis index based on the three factors; FIB-4, Fibrosis index based on the four factors; FibroQ, Fibro-quotient; GPR, $\mathrm{y}$-glutamyl transpeptidase/ platelet count ratio index; GUCl, Goteborg University Cirrhosis Index; PAPAS, Platelet count/ age/ ALP/ AFP/ AST index; King's score, Fibrosis index based on the four factors; Forns index, Platelet count/ $\gamma$-glutamyl transpeptidase/ age/ cholesterol index. 


\section{Determining the cut-off value of variables}

The ROC curve of PLT showed that $114 \times 10^{9} / \mathrm{L}$ was an optimum cut-off value, and the sensitivity plus specificity correspondingly was the maximal. Among these models, only CDS (AUC $=0.665,95 \% \mathrm{Cl}: 0.559-0.760$ ), APRI $\quad(A U C=0.632,95 \% \mathrm{Cl}: 0.525-0.730)$, King's score $(A \cup C=0.648,95 \% \mathrm{Cl}: 0.542-0.745)$, Lok index (AUC $=0.647,95 \% \mathrm{Cl}: 0.540-0.744)$, FIB-4 (AUC $=0.658,95 \% \mathrm{Cl}: 0.552-0.754)$, FibroQ (AUC $=0.661$, $95 \% \mathrm{Cl}: 0.555-0.756$ ) and $\mathrm{GUCl}$ (AUC $=0.630,95 \% \mathrm{Cl}$ : 0.523-0.728) were significant indicators for determining prognosis $(P<0.05)$ (Table 4$)$.

Table 4 - Comparison of the AUC between PLT-based prognostic scores.

\begin{tabular}{|c|c|c|c|c|}
\hline Variable & AUC & $95 \% \mathrm{CI}$ & Cut-off value & P-value \\
\hline PLT & 0.605 & $0.498-0.705$ & $114 \times 10^{9}$ & 0.08 \\
\hline API & 0.608 & $0.500-0.708$ & 4 & 0.07 \\
\hline CDS & 0.665 & $0.559-0.760$ & 6 & 0.003 \\
\hline APRI & 0.632 & $0.525-0.730$ & 1.36 & 0.029 \\
\hline King's score & 0.648 & $0.542-0.745$ & 31.31 & 0.013 \\
\hline Lok index & 0.647 & $0.540-0.744$ & 0.75 & 0.013 \\
\hline $\mathrm{P} 2 / \mathrm{MS}$ & 0.579 & $0.472-0.681$ & 42.46 & 0.19 \\
\hline PAPAS & 0.607 & $0.500-0.707$ & 4.36 & 0.07 \\
\hline PSR & 0.590 & $0.482-0.691$ & 1.04 & 0.14 \\
\hline S-index & 0.541 & $0.434-0.645$ & 0.64 & 0.5 \\
\hline FIB-4 & 0.658 & $0.552-0.754$ & 5.64 & 0.008 \\
\hline FibroQ & 0.661 & $0.555-0.756$ & 7.06 & 0.006 \\
\hline GUCI & 0.630 & $0.523-0.728$ & 60.21 & 0.03 \\
\hline GPR & 0.526 & $0.419-0.631$ & 0.87 & 0.7 \\
\hline APGA & 0.602 & $0.494-0.702$ & 32.03 & 0.09 \\
\hline Forn index & 0.602 & 0.494-0.702 & 10.57 & 0.10 \\
\hline
\end{tabular}

AUC, area under the curve; $\mathrm{Cl}$, confidence interval; PLT, platelet count; API, Age/ platelet count index; CDS, Cirrhosis discriminant score; APRI, Aspartate aminotransferase/ platelet count ratio index; King's score, Fibrosis index based on the four factors; Lok-index, Fibrosis index based on the three factors; P2/MS, monocyte fraction/ segmented neutrophil fraction/ platelet count index; PAPAS, Platelet count/ age/ ALP/ AFP/ AST index; PSR, platelet count/ spleen diameter(mm) ratio index; S-index, $y$-glutamyl transpeptidase/ platelet count/ serum albumin index; FIB-4, Fibrosis index based on the four factors; FibroQ, Fibro-quotient; GUCl, Goteburg University Cirrhosis Index; GPR, $\gamma$-glutamyl transpeptidase/ platelet count ratio index; APGA, Aspartateaminotransferase/ platelet count/ $\gamma$-glutamyl transpeptidase/ alphafetoprotein index; Forns index, Platelet count/ $\gamma$-glutamyl transpeptidase/ age/ cholesterol index.

\section{Survival and prognostic factors}

The median time of follow-up was 32.5 (range 1-73) months. Fifty-two (56.5\%) patients were alive at the end of the follow-up period, and $40(43.5 \%)$ patients had died. The 1-year, 3-year and 5-year overall survival rates were $83.5 \%, 64.5 \%$, and $40.2 \%$, respectively.

According to the optimum cut-off value determined by The ROC curve, the continuous variables of plateletbased scores were divided into two categories. The relationship between the platelet-based prognostic scores and overall survival is shown in Figure 1. The APGA $>32.03$, APRI $>1.36$, FIB-4>5.64, FibroQ $>7.06$, GUCl $>60.21$, King's score $>31.31$, Lok index $>0.75$ and PAPAS $>4.36$ were associated with a reduced overall survival (all $P<0.05$ ). Meanwhile, a log-rank analysis demonstrated that patients with cirrhosis, multiple tumor, vascular cancer embolus, AFP> $8.54 \mathrm{ng} / \mathrm{mL}$, ALP $>146 \mathrm{U} / \mathrm{L}$ and APTT $>28.1 \mathrm{~s}$ had a reduced probability of postoperative survival (Table 5 ). 

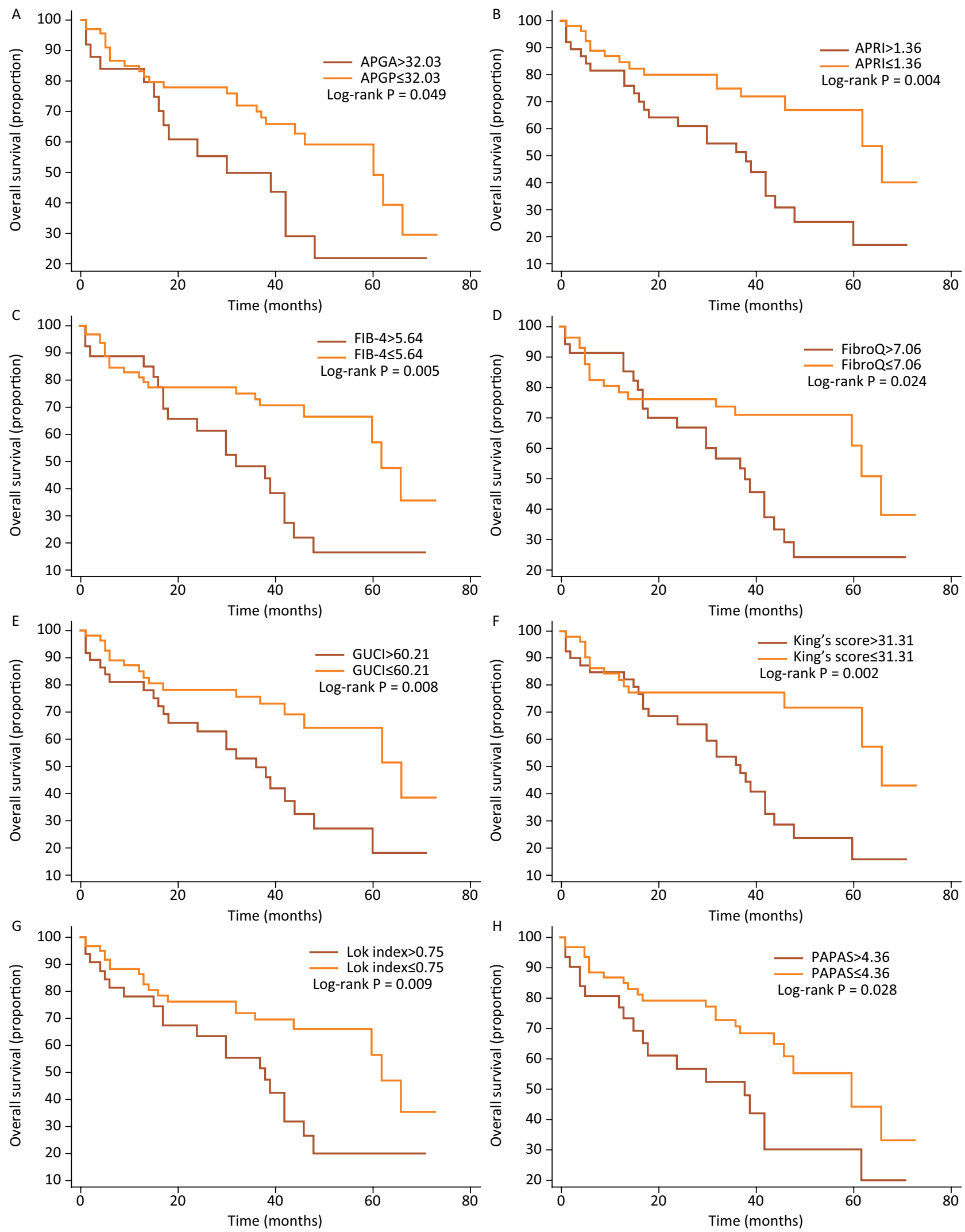

Figure 1 - Overall survival. Kaplan-Meier curves of patients stratified according to A) APGA, B) APRI, C) FIB-4, D) FibroQ, E) GUCl, F) King's score, G) Lok index and H) PAPAS. 
Table 5 - Log-rank test of possible clinical factors associating with survival status.

\begin{tabular}{|c|c|}
\hline Variable & P value \\
\hline Sex (male/female) & 0.396 \\
\hline Age $(\leq 52 Y />52 Y)$ & 0.112 \\
\hline Cirrhosis & 0.049 \\
\hline HBV (Yes/No) & 0.171 \\
\hline Ascites (Yes/No) & 0.337 \\
\hline Polycythemia (Yes/No) & 0.573 \\
\hline Number of tumors (single/multiple) & 0.040 \\
\hline Vascular cancer embolus (Yes/No) & $<0.001$ \\
\hline $\operatorname{AFP}(\leq 8.54 \mathrm{ng} / \mathrm{ml} />8.54 \mathrm{ng} / \mathrm{ml})$ & 0.038 \\
\hline $\operatorname{ALT}(\leq 98 \mathrm{U} / \mathrm{L} />98 \mathrm{U} / \mathrm{L})$ & 0.316 \\
\hline $\operatorname{AST}(\leq 38 \mathrm{U} / \mathrm{L} />38 \mathrm{U} / \mathrm{L})$ & 0.058 \\
\hline $\operatorname{ALP}(\leq 146 \mathrm{U} / \mathrm{L} />146 \mathrm{U} / \mathrm{L})$ & 0.016 \\
\hline $\mathrm{GGT}(\leq 177 \mathrm{U} / \mathrm{L} />177 \mathrm{U} / \mathrm{L})$ & 0.415 \\
\hline $\operatorname{ALB}(\leq 40 \mathrm{~g} / \mathrm{L} />40 \mathrm{~g} / \mathrm{L})$ & 0.105 \\
\hline $\operatorname{PT}(\leq 13.3 s />13.3 s)$ & 0.107 \\
\hline $\mathrm{APTT}(\leq 28.1 \mathrm{~s} />28.1 \mathrm{~s})$ & 0.001 \\
\hline $\operatorname{INR}(\leq 1.11 />1.11)$ & 0.131 \\
\hline Diameter of spleen $(\leq 95.3 \mathrm{~mm} />95.3 \mathrm{~mm})$ & 0.082 \\
\hline $\mathrm{PLT} \times 10^{9}(\leq 114 />114)$ & 0.132 \\
\hline $\operatorname{APGA}(\leq 32.03 />32.03)$ & 0.049 \\
\hline $\operatorname{API}(\leq 4 />4)$ & 0.148 \\
\hline $\operatorname{APRI}(\leq 1.36 />1.36)$ & 0.004 \\
\hline $\operatorname{CDS}(\leq 6 />6)$ & 0.088 \\
\hline FIB- $4(\leq 5.64 />5.64)$ & 0.005 \\
\hline FibroQ $(\leq 7.06 />7.06)$ & 0.024 \\
\hline Forn index $(\leq 10.57 />10.57)$ & 0.162 \\
\hline $\operatorname{GPR}(\leq 0.87 />0.87)$ & 0.056 \\
\hline $\operatorname{GUCI}(\leq 60.21 />60.21)$ & 0.008 \\
\hline King's score $(\leq 31.31 />31.31)$ & 0.002 \\
\hline Lok index $(\leq 0.75 />0.75)$ & 0.009 \\
\hline $\mathrm{P} 2 / \mathrm{MS}(\leq 42.46 />42.46)$ & 0.126 \\
\hline PAPAS $(\leq 4.36 />4.36)$ & 0.028 \\
\hline $\operatorname{PSR}(\leq 1.04 />1.04)$ & 0.193 \\
\hline S-index $(\leq 0.64 />0.64)$ & 0.200 \\
\hline Pohl score(negative/positive) & 0.166 \\
\hline AARP(negative/positive) & 0.914 \\
\hline
\end{tabular}

AFP, alpha fetoprotein; ALT, alanine transaminase; AST, aspartate amino transferase; ALP, alkaline phosphatase; GGT, gamma-glutamyl transpeptidase; ALB, albumin; PT, prothrombin time; APTT, activated partial thromboplastin time; INR, international normalized ratio; . PLT, platelet count; APGA, Aspartateaminotransferase/ platelet count/ $\gamma$-glutamyl transpeptidase/ alphafetoprotein index; API, Age/ platelet count index; APRI, Aspartate aminotransferase/ platelet count ratio index; CDS, Cirrhosis discriminant score; FIB-4, Fibrosis index based on the four factors; FibroQ, Fibro-quotient; Forns index, Platelet count/ $\gamma$-glutamyl transpeptidase/ age/ cholesterol index; GPR, $y$-glutamyl transpeptidase/ platelet count ratio index; $\mathrm{GUCl}$, Goteborg University Cirrhosis Index; King's score, Fibrosis index based on the four factors; Lok-index, Fibrosis index based on the three factors; P2/MS, monocyte fraction/ segmented neutrophil fraction/ platelet count index; PAPAS, Platelet count/ age/ ALP/ AFP/ AST index; PSR, platelet count/ spleen diameter $(\mathrm{mm})$ ratio index; S-index, $\gamma$-glutamyl transpeptidase/ platelet count/ serum albumin index; . AAR, Aspartate aminotransferase/alanine aminotransferase ratio; PLT, Platelet count; AARP, AAR-platelet count score.
Multivariate analysis of clinical characteristic variables expressed as binary variables showed that the number of tumors, ALP and APTT were independent risk factors for overall survival. Among the noninvasive platelet-based scores, multivariate analysis for the 17 predictive factors and platelet count revealed that APGA (HR 3.213, 95\% Cl 1.089-9.477, $\mathrm{P}=0.034$ ) was independently associated with overall survival. The other 16 platelet-unrelated scores and platelet count, API, APRI, CDS, FIB-4, FibroQ, Forn index, GPR, GUCl, King's score, Lok index, P2/MS, PAPAS, PSR, S-index, Pohl score and AARP, were not independent risk factors for overall survival. (Table 6).

Table 6 - Results of multivariate analyses for survival status.

\begin{tabular}{lccc}
\hline Variable & OR & $\mathbf{9 5 \%} \mathbf{C I}$ & $\mathbf{P}$ \\
\hline Cirrhosis & 1.272 & $0.558-2.901$ & 0.568 \\
Number of tumors & 0.52 & $0.258-1.048$ & 0.067 \\
Vascular cancer & 0.219 & $0.084-0.570$ & 0.002 \\
embolus & 0.513 & $0.176-1.496$ & 0.221 \\
AFP & 0.406 & $0.179-0.922$ & 0.031 \\
ALP & 0.088 & $0.021-0.371$ & 0.001 \\
APTT & 3.213 & $1.089-9.477$ & 0.034 \\
APGA & 0.558 & $0.094-3.297$ & 0.52 \\
APRI & 0.500 & $0.104-2.414$ & 0.388 \\
FIB-4 & 2.037 & $0.440-9.431$ & 0.363 \\
FibroQ & 1.472 & $0.245-8.833$ & 0.672 \\
GUCI & 0.460 & $0.116-1.824$ & 0.269 \\
King's score & 0.666 & $0.278-1.591$ & 0.360 \\
Lok index & 0.923 & $0.344-2.481$ & 0.874 \\
PAPAS & O. &
\end{tabular}

$\mathrm{OR}$, odds ratio; $\mathrm{Cl}$, confidence interval; $\mathrm{AFP}$, alpha

fetoprotein; ALP, alkaline phosphatase; APTT, activated partial thromboplastin time; APGA, Aspartateaminotransferase/ platelet count/ -glutamyl transpeptidase/ alphafetoprotein index; APRI, Aspartate aminotransferase/ platelet count ratio index; FIB-4, Fibrosis index based on the four factors; FibroQ, Fibro-quotient; GUCl, Goteborg University Cirrhosis Index; King's score, Fibrosis index based on the four factors; Lok-index, Fibrosis index based on the three factors; PAPAS, Platelet count/ age/ ALP/ AFP/ AST index.

\section{- Discussion}

The ultimate goal of a treatment for malignant hepatic tumors was to prolong survival time by curative therapy. TACE and resection were the most frequent preferred treatments for advanced hepatocellular 
carcinoma in clinical practice ${ }^{28}$. When patients couldn't perform radical resection, TACE treatment might be a good choice for these patients. For example, a single central lesion that located between the portal vein and the inferior vena cava might be an indication of TACE treatment, the reason was the difficulty of radical resection or the insufficient volume of residual liver. However, indications for TACE were not applicable to all candidates such as Child-Pugh $C$ grade. For Child-Pugh $C$ grade, it could be restored to Child-Pugh A or B grade after adjustment, and TACE treatment could still be performed. This didn't mean that all patients with advanced HCC could benefit from TACE treatment. The variation in prognosis was significant according to the number of prognostic risk factors, with median survival time from 5.5 months to 21.4 months $^{29}$. Thus, it was crucial to determine the predisposing factors for prognosis and improve them before treatment. The roles of platelet count and platelet-based score models for assessing such risk factors were emphasized in this retrospective analysis of 92 patients. The results confirmed that the number of tumors, ALP and APTT were independent prognostic factors of overall survival. We also determined APGA as independent predictors of overall survival after TACE therapy.

Platelet usually released all kinds of cytokines to participate in the inflammatory response, such as platelet-derived growth factors and transforming growth factor $\beta$. Platelets also transport these substances to specific positions, platelet played an important role in angiogenesis, wound healing, liver regeneration and so on 7 . Most liver cancers were accompanied with cirrhosis, which was mainly caused by chronic hepatitis. When liver cancer forms, cancer cells could disorder the balance of the blood coagulation system by producing high levels of blood coagulation factors. Besides, the disorders of the coagulation system promoted excessive platelet activation. The activated platelets could provide the procoagulant surface to induce cancer-related gather. Meanwhile, the platelets were recruited to surround tumor cells, which protected them from the body's immune detection and promoted the growth and metastasis of the cancer ${ }^{30,31}$. It could also promote the release of active medium of platelets and increase the permeability of blood vessels to achieve the goal of cancer proliferation ${ }^{32}$. Therefore, these theoretical backgrounds had led to the proposal of several plateletbased prognostic scores in patients with cancer over the last 10 years.

Several studies had demonstrated that PLT was associated with hepatocellular carcinoma, and several platelet-based models had been determined as predictors of cancer formation ${ }^{33}$. Tamaki et al reported that the FIB-4>3.25 independently increased the risk of developing hepatocellular carcinoma by a factor of $1.7^{34}$. Amano et al. $^{35}$ demonstrated that low-level PLT was associated with a higher risk of recurrence in hepatocellular carcinoma. Many prognostic models of malignant hepatic tumors had been reported, but which model was more suitable for predicting the prognosis of malignant hepatic tumors was still inconclusive. Moreover, reliable indices to predict long-term prognosis after TACE were still scarce. To improve the outcomes of advanced malignant hepatic tumor patients after TACE, it was necessary to illustrate the mechanism of oncogenesis and explore the clinically crucial risk factors associated with longterm prognosis, which might be the potential therapy targeting except for radical surgery. In this study, we believed that platelet count and platelet-based scores might be potential therapeutic targets and the score of APGA>32.03 was an independent risk factor associated with poor overall survival for patients with malignant hepatic tumor undergoing TACE. Therefore, the APGA index would be best applicable in predicting prognosis, and overall survival also could be predicted according to APGA score and corresponding preoperative adjustment could be made.

The result was consistent with that of Fung et al, the APGA score could predict hepatitis, cirrhosis, even liver cancer $^{21}$. As can be seen from Figure $1 \mathrm{~A}$, the higher the score, the lower the survival rate after TACE. According to the calculation formula of APGA from Table 1, the higher the platelet count, the higher the score. The platelet count was not an independent risk factor for prognosis but the APGA score was; the reason for this result was the combined effect of PLT, AST, GGT and AFP. Although the AUC value of APGA score (AUC=0.602) was not the largest in Table 4 and the $P$ value is greater than 0.05 , it did not mean that the APGA score was worthless. The main purpose of table 4 was to obtain the optimal cutoff value by ROC curve, rather than to compare various platelet models to diagnose patient outcome events. As long as the AUC value of APGA was more than 0.5, we could still perform further analysis and research on the APGA model.

This study was an exploratory analysis of clinical data including patients who underwent TACE treatment and obtained different prognosis backgrounds. In this study, in addition to the conclusion that APGA was an independent risk factor affecting the prognosis after TACE, the number of tumors, ALP and APTT were also independent risk factors. Chan et al pointed out that the number of tumors was a key parameter for early recurrence of 
$\mathrm{HCC}^{36}$. Our results in line with Chan, multi-nodulose tumor was associated with poor prognosis after TACE. We believed that multi-nodulose tumor might have undetected micro lesions, which were easy to recurrence after surgery. Coagulation disorders and abnormal liver metabolism were usual complication in liver diseases, especially advanced tumors. Our results also suggested that ALP and APTT were independent risk factors for prognosis after TACE. Therefore, we can also predict the prognosis of TACE by ALP and APTT preoperatively.

A potential limitation of this study was that was a retrospective, single-center study. Therefore, a largescale prospective study was needed to validate the result. These scores were not compared with plateletunrelated indices and stratification analysis of patients was not used to determine whether results of plateletbased models were affected.

This study was the first to explore the performances of 17 platelet-based scores for preoperative detecting overall survival after TACE in malignant hepatic tumors. Furthermore, we could make appropriate preoperative adjustment according to the score of the optimum platelet-based model, so as to improve the survival rate and prolong survival time after TACE. Additionally, all selected platelet-based models had been reported and accepted that have been repeatedly validated in corresponding liver diseases. Taken together, the study showed that APGA might be an effective tool to assess postoperative prognosis in patients with malignant hepatic tumors, especially overall survival. As the prognostic index was noninvasive, inexpensive, and easy to count, these findings would be meaningful for surgeons or interventional radiologists assessing postoperative overall survival in malignant hepatic tumors.

\section{- Conclusion}

The APGA, a platelet-based prognostic score, was an independent marker of prognosis in patients with malignant hepatic tumors after TACE and was superior to the other platelet-based prognostic scores in terms of prognostic ability.

\section{- References}

1. Bray F, Ferlay J, Soerjomataram I, Siegel RL, Torre LA, Jemal A. Global cancer statistics 2018: GLOBOCAN estimates of incidence and mortality worldwide for 36 cancers in 185 countries. CA Cancer J Clin. 2018;68(6):394-424. doi: 10.3322/caac.21492.
2. Buonaguro L, Petrizzo A, Tagliamonte M, Tornesello ML, Buonaguro FM. Challenges in cancer vaccine development for hepatocellular carcinoma. J Hepatol. 2013;59(4):897903. doi: 10.1016/j.jhep.2013.05.031.

3. Llovet JM, Bru C, Bruix J. Prognosis of hepatocellular carcinoma: the BCLC staging classification. Semin Liver Dis. 1999;19(03):329-38. doi: 10.1055/s-2007-1007122.

4. Metussin A, Patanwala I, Cross TJ. Partial hepatectomy vs. transcatheter arterial chemoembolization for resectable multiple hepatocellular carcinoma beyond Milan criteria: a RCT. J Hepatol. 2015;62(3):747-8. doi: 10.1016/j. jhep.2014.08.057.

5. Mezouar S, Frère $C$, Darbousset $R$, Mege $D$, Crescence L, Dignat-George F, Panicot-Dubois L, Dubois C. Role of platelets in cancer and cancer-associated thrombosis: experimental and clinical evidences. Thromb Res. 2016;139: 65-76. doi: 10.1016/j.thromres.2016.01.006.

6. Walsh TG, Metharom P, Berndt MC. The functional role of platelets in the regulation of angiogenesis. Platelets. 2015;26(3):199-211. doi: 10.3109/09537104.2014.909022.

7. Kondo R, Nakashima O, Tanikawa K, Nomura Y, Kage M. Accumulation of platelets in the liver may be an important contributory factor to thrombocytopenia and liver fibrosis in chronic hepatitis C. J Gastroenterol. 2013;48(4):526-34. doi: 10.1007/s00535-012-0656-2.

8. Starlinger $P$, Assinger A, Haegele $S$, Wanek D, Zikeli $S$, Schauer D, Birner P, Fleischmann E, Gruenberger B, Brostjan C, Gruenberger T. Evidence for serotonin as a relevant inducer of liver regeneration after liver resection in humans. Hepatology. 2014;60(1):257-66. doi: 10.1002/ hep.26950.

9. Wai CT, Greenson JK, Fontana RJ, Kalbfleisch JD, Marrero JA, Conjeevaram HS, Lok SF. A simple noninvasive index can predict both significant fibrosis and cirrhosis in patients with chronic hepatitis C. Hepatology. 2010;38(2):518-26. doi: 10.1053/jhep.2003.50346.

10. Cross TJ, Rizzi P, Berry PA, Bruce M, Portmann B, Harrison PM. King's Score: an accurate marker of cirrhosis in chronic hepatitis C. Eur J Gastroenterol Hepatol. 2009;21(7):730-8. doi: 10.1097/MEG.0b013e32830dfcb3.

11. Hsieh YY, Tung SY, Lee IL, Lee K, Shen CH, Wei KL, Chang TS, Chuang CS, Wu CS, Lin YH. FibroQ: an easy and useful noninvasive test for predicting liver fibrosis in patients with chronic viral hepatitis. Chang Gung Med J. 2009;32(06):61422. PMID: 20035640.

12. Toyoda H, Kumada T, Tada T, Kaneoka Y, Maeda A. A laboratory marker, FIB-4 index, as a predictor for longterm outcomes of hepatocellular carcinoma patients after curative hepatic resection. Surgery. 2015;157(4):699-707. doi: 10.1016/j.surg.2014.10.022.

13. Hussain SA, Ferry DR, El-Gazzaz G, Mirza DF, James ND, Mcmaster P, Kerr DJ. Hepatocellular carcinoma. Ann Oncol. 2001;12(2):161-72. PMID: 11300318.

14. Pohl A, Behling C, Oliver D, Kilani M, Monson P, Hassanein T. Serum aminotransferase levels and platelet counts as predictors of degree of fibrosis in chronic hepatitis $C$ virus infection. Am J Gastroenterol. 2001;96(11):3142-6. doi: 10.1111/j.1572-0241.2001.05268.x. 
15. Lee IC, Chan CC, Huang YH, Huo TI, Chu CJ, Lai CR, Lee PC, Su CW, Hung HH, Wu JC, Lin HC, Lee SD. Comparative analysis of noninvasive models to predict early liver fibrosis in hepatitis B e Antigen-negative Chronic Hepatitis B. J Clin Gastroenterol. 2011;45(3):278-85. doi: 10.1097/ mcg.0b013e3181dd5357.

16. Poynard T, Bedossa P. Age and platelet count: a simple index for predicting the presence of histological lesions in patients with antibodies to hepatitis $C$ virus. METAVIR and CLINIVIR Cooperative Study Groups. J Viral Hepat. 2010;4(3):199208. doi: 10.1046/j.1365-2893.1997.00141.x.

17. Bonacini M, Hadi G, Govindarajan S, Lindsay KL. Utility of a discriminant score for diagnosing advanced fibrosis or cirrhosis in patients with chronic hepatitis $C$ virus infection. Am J Gastroenterol. 1997;92(8):1302-4. doi: 10.1046/j.1365-2036.1997.00205.x.

18. Sterling RK, Eduardo L, Nathan C, Ricard S, Mendes Cassia C, Julio M, Mark SS, Torriani FJ, Dieterich DT, Thomas DL. Development of a simple noninvasive index to predict significant fibrosis in patients with HIV/HCV coinfection. Hepatology. 2010;43(6):1317-25. doi: 10.1002/hep.21178.

19. Asfghany L. Predicting cirrhosis in patients with hepatitis $C$ based on standard laboratoiy tests: results of the HALT-C cohort. Hepatology. 2010;42(2):282-92. doi: 10.1002/ hep. 20772.

20. Islam S. Cirrhosis in hepatitis $C$ virus- infected patients can be excluded using an index of standard biochemical serum markers. Scand J Gastroenterol. 2005;40(7):867-72. doi: 10.1080/00365520510015674.

21. Fung J, Lai CL, Fong DY, Yuen JC, Wong DK, Yuen MF. Correlation of liver biochemistry with liver stiffness in chronic hepatitis $B$ and development of a predictive model for liver fibrosis. Liver Int. 2010;28(10):1408-16. doi: 10.1111/j.1478-3231.2008.01784.x.

22. Seto WK, Lee CF, Lai CL, Ip PP, Fong DY, Fung J, Wong DK, Yuen MF. A new model using routinely available clinical parameters to predict significant liver fibrosis in chronic hepatitis B. Plos One. 2011;6(8):e23077. doi: 10.1371/ journal.pone.0023077.

23. Park YE, Kim BK, Park JY, Kim DY, Ahn SH, Han KH, Han $\mathrm{S}$, Jeon MY, Heo JY, Song K, Kim SU. Gamma-glutamyl transpeptidase-to-platelet ratio is an independent predictor of hepatitis B virus-related liver cancer. J Gastroenterol Hepatol. 2017;32(6):1221-9. doi: 10.1016/ S0168-8278(16)00490-6.

24. Zhou K, Gao CF, Zhao YP, Liu HL, Zheng RD, Xian JC, Xu HT, Mao YM, Zeng MD, Lu LG. Simpler score of routine laboratory tests predicts liver fibrosis in patients with chronic hepatitis B. J Gastroenterol Hepatol. 2010;25(9):1569-77. doi: 10.1111/j.1440-1746.2010.06383.x.

25. Testa R, Testa E, Giannini E, Borro P, Milazzo S, Isola L, Ceppa $\mathrm{P}$, Lantieri PB, Risso D. Noninvasive ratio indexes to evaluate fibrosis staging in chronic hepatitis C: role of platelet count/ spleen diameter ratio index. J Intern Med. 2010;260(2):14250. doi: 10.1111/j.1365-2796.2006.01673.x.
26. Lee $\mathrm{JH}$, Yoon $\mathrm{JH}$, Lee $\mathrm{CH}$, Myung $\mathrm{SJ}$, Keam B, Kim BH, Chung GE, Kim W, Kim YJ, Jang JJ, Lee HS. Complete blood count reflects the degree of oesophageal varices and liver fibrosis in virus-related chronic liver disease patients. J Viral Hepat. 2009;16(6):444-52. doi: 10.1111/j.13652893.2009.01091.x.

27. Güzelbulut F, Çetınkaya ZA, Sezıklı M, Yaşar B, Ozkara S, Övünç AO. AST-platelet ratio index, Forns index and FIB4 in the prediction of significant fibrosis and cirrhosis in patients with chronic hepatitis C. Turk J Gastroenterol. 2011;22(3):279-85. doi: 10.4318/tjg.2011.0213.

28. Park JW, Chen M, Colombo M, Roberts LR, Schwartz $M$, Chen PJ, Kudo M, Johnson P, Wagner S, Orsini LS. Global patterns of hepatocellular carcinoma management from diagnosis to death: the BRIDGE Study. Liver Int. 2015;35(9):2155-66. doi: 10.1111/liv.12818.

29. Le Y, Shen JX, Zhang YF, He MK, Kan A, Chen HL, Yu ZS, Li QJ, Shi M. Transarterial chemoembolization related to good survival for selected patients with advanced hepatocellular carcinoma. J Cancer. 2019;10(3):665-71. PMID: 30719164.

30. Myriam L, Shahinoor B, Hynes RO. Direct signaling between platelets and cancer cells induces an epithelial-mesenchymallike transition and promotes metastasis. Cancer Cell. 2011;20(5):576-90. doi: 10.1016/j.ccr.2011.09.009.

31. Bambace NM, Holmes CE. The platelet contribution to cancer progression. J Thromb Haemost. 2011;9(2):237-49. doi: 10.1111/j.1538-7836.2010.04131.x.

32. Pang $Q$, Qu K, Bi JB, Liu SS, Zhang JY, Song SD, Lin T, Xu $\mathrm{XS}$, Wan $\mathrm{Y}$, Tai $\mathrm{MH}$. Thrombocytopenia for prediction of hepatocellular carcinoma recurrence: Systematic review and meta-analysis. World J Gastroenterol. 2015;21(25):7895-906. doi: 10.3748/wjg.v21.i25.7895.

33. Carr BI, Guerra V. Thrombocytosis and hepatocellular carcinoma. Dig Dis Sci. 2013;58(6):1790-6. doi: 10.1007/ s10620-012-2527-3.

34. Tamaki N, Kurosaki M, Matsuda S, Muraoka M, Yasui $Y$, Suzuki S, Hosokawa T, Ueda K, Tsuchiya K, Nakanishi H, Itakura J, Takahashi Y, Asahina Y, Izumi N. Non-invasive prediction of hepatocellular carcinoma development using serum fibrosis marker in chronic hepatitis C patients. J Gastroenter. 2014;49(11):1495-503. doi: 10.1007/s00535013-0914-y.

35. Amano $H$, Tashiro $H$, Oshita A, Kobayashi T, Tanimoto $\mathrm{Y}$, Kuroda S, Tazawa H, Itamoto T, Asahara T, Ohdan $\mathrm{H}$. Significance of platelet count in the outcomes of hepatectomized patients with hepatocellular carcinoma exceeding the Milan criteria. J Gastrointest Surg. 2011;15(7):1173-81. doi: 10.1007/s11605-011-1538-2.

36. Chan AWH, Zhong J, Berhane S, Toyoda H, Cucchetti A, Shi K, Tada T, Chong CC, Xiang BD, Li LQ, Lai PBS, Mazzaferro V, García-Fiñana M, Kudo M, Kumada T, Roayaie S, Johnson PJ. Development of pre and post-operative models to predict early recurrence of hepatocellular carcinoma after surgical resection. J Hepatol. 2018;69(6):1284-93. doi: 10.1016/j. jhep.2018.08.027. 


\section{Correspondence:}

Ying Zhou

Department of Hepatopancreatobiliary Surgery

The Affiliated Hospital, Qinghai University

Xining 810000 China

Phone: +869716230701

17697227511@163.com

Received: Mar 09, 2019

Review: May 06, 2019

Accepted: June 08, 2019

\section{Conflict of interest: none}

Financial source: Science and Technology Department of Qinghai province (2016-SF-A5)

This is an Open Access article distributed under the terms of the Creative Commons Attribution License, which permits unrestricted use, distribution, and reproduction in any medium, provided the original work is properly cited.

1 Research performed at Department of Hepatopancreatobiliary Surgery, The Affiliated Hospital, Qinghai University, Xining, China. 